\title{
Prognostic analysis of hepatocellular carcinoma on the background of liver cirrhosis via contrast-enhanced ultrasound and pathology
}

\author{
YUKAI HE ${ }^{1}$, FENGHUA LIU ${ }^{1}$, SHAOFENG MOU ${ }^{2}$, QINGYAN LI $^{1}$ and SIKUI WANG ${ }^{1}$ \\ Departments of ${ }^{1}$ Infectious Disease and ${ }^{2}$ Otorhinolaryngology, Liaocheng People's Hospital, \\ Liaocheng, Shandong 252000, P.R. China
}

Received July 27, 2017; Accepted December 5, 2017

DOI: $10.3892 / 01.2018 .7792$

\begin{abstract}
The aim of this study was to analyze the correlation between the quantitative parameters of contrast-enhancement ultrasound for primary hepatocellular carcinoma (HCC) and biological manifestations of tumor (Ki-67), and to explore the related risk factors of primary hepatocellular carcinoma, so as to provide the theoretical basis for the further study on contrast-enhancement ultrasound manifestations, clinical features and prognosis of HCC. The patients with HCC confirmed by operation or puncture were collected, and those with the background of liver cirrhosis and immunohistochemical staining for tumor sample sections were selected. H\&E staining sections of pathological tissues of tumor samples were observed, whether there was any microvessel invasion (MVI) was recorded, the microvessel density (MVD) was counted and the recurrence situations after liver cancer operation was followed up. The change in size of tumor at arterial phase in contrast-enhancement ultrasound, enhancement mode and form at arterial phase, and whether there were tortuous vessels inside or not, and the enhancement intensity, extinction time and extinction intensity at portal phase were observed. The relationship between the parameters of contrastenhancement ultrasound and Ki-67, AFP, MVD, MVI, tissue differentiation degree of tumor samples and recurrence was analyzed. Under the background of liver cirrhosis, there were significant differences in different enhancement modes and quantification parameters of contrast-enhancement ultrasound for HCC with different expression of Ki-67. Those with obvious tumor enlargement, inhomogeneous enhancement at arterial phase and irregular enhancement form at arterial phase after contrast-enhancement ultrasound had a high inci-
\end{abstract}

Correspondence to: Dr Qingyan Li, Department of Infectious Disease, Liaocheng People's Hospital, 67 Dongchang-xi Road, Liaocheng, Shandong 252000, P.R. China

E-mail: qingyanlill@126.com

Key words: HCC, contrast-enhancement ultrasound, pathology, $\mathrm{Ki}-67$, prognosis dence of positive Ki-67 and a high early recurrence rate. The inhomogeneous enhancement at arterial phase might predict the proliferative activity and recurrence time of tumor cells; irregular enhancement form at arterial phase might indicate tumor MVI; and the low enhancement of tumor at portal phase may predict a lower degree of tissue differentiation, a higher tumor malignancy and poor prognosis. The incidence of positive $\mathrm{Ki}-67$ under the background of liver cirrhosis is high, indicating poor prognosis. The enhancement mode and parameters of contrast-enhancement ultrasound for HCC may help evaluate the clinical biological manifestations of HCC and predict the postoperative recurrence of HCC.

\section{Introduction}

Primary liver cancer is one of the most common malignant tumors in the world, among which hepatocellular carcinoma (HCC) accounts for approximately $70-85 \%$ (1). Surgical operation, local ablation, radiotherapy and chemotherapy provide opportunities to cure or prolong the survival time of patients. However, the 5-year recurrence rate after primary liver cancer operation is as high as $45-60 \%$, seriously affecting the prognosis of patients with liver cancer (2-4). Therefore, searching for the indexes for evaluating the poor prognosis of $\mathrm{HCC}$, such as proliferative activity and early recurrence, is of significance for the tumor staging, selection of treatment plan and prognosis.

$\mathrm{Ki}-67$ is a kind of nuclear antibody $\mathrm{Ki}-67$ protein, mainly existing in the dense fibrillar components of nucleoli and cortices, which is a reliable index of detecting the tumor cell proliferative activity (5). The biological manifestations of tumor are closely related to its proliferative activity. The higher the expression of Ki-67 is, the faster the tumor cell proliferation will be and the higher the probability of metastasis will also be $(6,7)$. However, there are few studies on the correlation between contrast-enhancement ultrasound of HCC and Ki-67 immunohistochemistry and other prognostic factors.

Compared with enhancement MRI/CT, PET and other examinations, contrast-enhancement ultrasound (CEUS) is characterized by the convenient and efficient application, wide indications and no radiation, and it can show the whole process of contrast enhancement for tumor and liver parenchyma in a real-time and dynamic manner, which not only 
improves the value of diagnosis and differential diagnosis, but also indirectly reflects some biological manifestations of tumors (8-10). In the field of contrast-enhancement ultrasound, there are some scholars who use special computer software to conduct the quantization parameter analysis for CEUS images of HCC, establish the peak intensity curve, and study the relationship between CEUS parameters on the curve and microvessel density (MVD), microvessel invasion (MVI) and tumor tissue differentiation degree (11-14). These factors are associated with the recurrence of liver cancer and the survival time of liver cancer patients.

This study aimed to evaluate the relationship between the enhancement mode and parameters of CEUS for HCC and the prognostic factors and clinical recurrence, and evaluate the biological behavior and prognosis of liver cancer from the perspective of ultrasonography, so as to provide new noninvasive evaluation indexes of prognosis.

\section{Patients and methods}

Subjects. One hundred and eighty patients with HCC confirmed by operation or puncture were collected, and they all had the background of liver cirrhosis. The tumor samples received immunohistochemical Ki-67 and AFP-labeled staining. This study was approved by the Ethics Committee of Qianfoshan Hospital Affiliated to Shandong University (Jinan, China). Signed written informed consents were obtained from all participants before the study.

Pathological examination of $\mathrm{Ki}$-67. Each section was observed via 10 visual fields randomly selected under high-power microscope (x400), and the percentage of brown-stained cell nuclei every 100 cells was the percentage of Ki-67. Ki-67 was divided into Ki-67-positive group (>10\%) and the Ki-67negative group $(\leq 10 \%)$ according to the percentage of Ki-67.

Microvessel density, MVD. After the blood vessels in tumor tissues were stained by antigen-antibody reaction, the slide was glanced with low-power microscope $(\mathrm{x} 40-\mathrm{x} 100)$ to find the area with the highest blood vessel density. Then the stained blood vessels within the field were counted under high-power microscope (x400). The mean MVD of the patients included was calculated, and they were divided into the high-MVD group (greater than the mean) and low-MVD group (lower than the mean).

Microvessel invasion, MVI. Microvessel infiltration means that multiple or massed cancer cells can be found in venules in paracancer mesenchyme, obvious endothelial cells can be found around the lumen and red blood cells may appear within the lumen. Patients were divided into the MVI-positive group (MVI was found) and MVI-negative group (no MVI was found).

Contrast-enhancement ultrasound, CEUS. Color Doppler ultrasound was used to record the lesion site and size, internal echo and number, and Doppler blood flow signal; and then the contrast agent was injected under the radiography mode, and the timer and recording were started. The sum of two maximum orthogonal radial lines of lesion on the same section was taken as size of the tumor; according to the changes before and after CEUS, those greater than the mean of all change values were enrolled into the significant change group; otherwise, they were enrolled into the non-significant change group. The image at 10-30 sec after injection of contrast agent was taken as arterial phase. The images with peak intensity were observed; if there were low enhancement or no enhancement area in different sizes in the lesion, the enhancement mode was seen as the inhomogeneous enhancement. The irregular enhancement form of lesion was regarded as the irregular tumor form. Whether there were tortuous blood vessels in the enhancement process at arterial phase were observed. The images at 90-120 sec after injection of contrast agent were selected as the portal phase. In comparison with surrounding liver parenchyma, the extinction time of contrast agent was recorded, the enhancement intensity and regression degree of lesion at portal phase were observed.

Recurrence of liver cancer. If the focal hepatic lesions showed the typical manifestation of HCC or obvious extra hepatic metastasis in imaging examination for the first time, it was the recurrence. According to the first time of discovering recurrent lesion, it was divided into early recurrence ( $\leq 1$ year) and late recurrence ( $>1$ year).

Statistical analysis. All statistics were performed using SPSS 19.0 software (SPSS Inc., Chicago, IL, USA). t-test was performed for the comparison of mean value, Chi-square test for the significant difference between groups. Binary logistic regression analysis was used to evaluate the relationship between the parameters of ultrasound contrast and prognostic factors. $\mathrm{P}<0.05$ was considered to indicate a statistically significant difference.

\section{Results}

Ki-67/AFP immunohistochemistry. In this study, the clinical characteristics between Ki-67 positive group (Fig. 1) and Ki-67 negative group (Fig. 1), AFP positive group and AFP negative group were compared. There were no statistically significant differences in the age, sex, positive rate of hepatitis B serum marker and liver function Child-Pugh grade between the two groups $(\mathrm{P}>0.05)$; but the proportion of high-differentiated liver cancer in Ki-67 negative group was higher than that in Ki-67 positive group, and the proportion of low-differentiated liver cancer in $\mathrm{Ki}-67$ positive group was higher than that in Ki-67 negative group $(\mathrm{P}<0.05)$ (Table I).

Microvessel density (MVD). In this study, the mean MVD of patients was 63.40, and they were divided into the high-MVD group (greater than the mean) and low-MVD group (lower than the mean) (Fig. 2). There were no statistically significant differences in the age, sex, hepatitis B serum marker and liver function between high-MVD group and low-MVD group ( $\mathrm{P}>0.05)$.

Microvessel invasion (MVI). In this study, patients were divided into the MVI-positive group (MVI was found in para-cancer mesenchyme) (Fig. 3) and MVI-negative group (no MVI was found in para-cancer mesenchyme) (Fig. 3). There were no statistically significant differences in the age, sex, hepatitis B serum marker and liver function between MVI-positive group and MVI-negative group $(\mathrm{P}>0.05)$. 
Table I. Basic characteristics of Ki-67(+)/Ki-67(-) HCC subjects.

\begin{tabular}{|c|c|c|c|c|}
\hline Factors & $\mathrm{Ki}-67(+)$ & $\mathrm{Ki}-67(-)$ & Test value & P-value \\
\hline Sex (male \%) & $90.01 \%$ & $91.97 \%$ & $\chi^{2}=0.23$ & 0.57 \\
\hline Age & $45.90 \pm 8.76$ & $48.86 \pm 9.34$ & $\mathrm{t}=1.74$ & 0.23 \\
\hline Tumor tissue differentiation degree & & & $\chi^{2}=16.28$ & NS \\
\hline High-differentiated & $2.63 \%$ & $15.57 \%$ & & \\
\hline Medium-differentiated & $81.87 \%$ & $79.96 \%$ & & \\
\hline Low-differentiated & $15.50 \%$ & $4.47 \%$ & & \\
\hline Hepatitis B serum marker & & & $\chi^{2}=4.26$ & 0.08 \\
\hline Positive & 99 & 69 & & \\
\hline Negative & 6 & 6 & & \\
\hline Liver function Child-Pugh grade & & & $\chi^{2}=0.02$ & 0.88 \\
\hline Grade A & 96 & 70 & & \\
\hline Grade B & 9 & 5 & & \\
\hline
\end{tabular}

HCC, hepatocellular carcinoma; Ki-67(+), Ki-67-positive group; Ki-67(-), Ki-67-negative group. NS, not significant.

Table II. Comparison of CEUS parameters in Ki-67/AFP different HCC groups.

\begin{tabular}{|c|c|c|c|c|c|c|c|c|}
\hline Factors & $\mathrm{Ki}-67(+)$ & $\mathrm{Ki}-67(-)$ & $\chi^{2}$ test & P-value & $\mathrm{AFP}(+)$ & $\operatorname{AFP}(-)$ & $\chi^{2}$ test & P-value \\
\hline Change of tumor size & & & 6.15 & 0.01 & & & 2.31 & 0.10 \\
\hline Conspicuous & 46 & 20 & & & 40 & 26 & & \\
\hline Inconspicuous & 59 & 55 & & & 54 & 60 & & \\
\hline Enhancement mode at arterial phase & & & 6.57 & 0.00 & & & 0.13 & 1.13 \\
\hline Inhomogeneous & 79 & 34 & & & 59 & 55 & & \\
\hline Homogeneous & 26 & 41 & & & 35 & 31 & & \\
\hline Enhancement form at arterial phase & & & 7.34 & 0.02 & & & 0.98 & 0.56 \\
\hline Irregular & 49 & 21 & & & 40 & 31 & & \\
\hline Regular & 56 & 54 & & & 54 & 55 & & \\
\hline Tortuous vessels & & & 2.39 & 0.26 & & & 1.13 & 0.63 \\
\hline Yes & 44 & 24 & & & 33 & 36 & & \\
\hline No & 61 & 51 & & & 61 & 50 & & \\
\hline Enhancement intensity at portal phase & & & 7.43 & 0.04 & & & 1.77 & 0.46 \\
\hline Low intensity & 81 & 39 & & & 64 & 56 & & \\
\hline High or equal intensity & 24 & 36 & & & 30 & 30 & & \\
\hline Extinction speed at portal phase & & & 8.12 & 0.05 & & & 3.12 & 0.50 \\
\hline Fast & 55 & 24 & & & 39 & 41 & & \\
\hline Low & 50 & 51 & & & 55 & 45 & & \\
\hline Extinction extent at portal phase & & & 8.64 & 0.04 & & & 0.85 & 0.67 \\
\hline Obvious & 79 & 37 & & & 62 & 56 & & \\
\hline None or slight & 26 & 38 & & & 32 & 30 & & \\
\hline
\end{tabular}

HCC, hepatocellular carcinoma; Ki-67(+), Ki-67-positive group; Ki-67(-), Ki-67-negative group.

Relationship between CEUS parameters vs. pathologic histology. The incidence of positive Ki-67 was high for those cases with obvious change in tumor size, inhomogeneous enhancement and irregular enhancement form of tumor at arterial phase, and low enhancement and fast extinction at portal phase after CEUS ( $\mathrm{P}<0.05$, Table II). There was no significant correlation between the change in tumor size, enhancement mode and form of tumor at arterial phase and 
Table III. Comparison of CEUS parameters in MVD/MVI different HCC groups.

\begin{tabular}{|c|c|c|c|c|c|c|c|c|}
\hline Factors & H-MVD & L-MVD & $\chi^{2}$ test & P-value & $\operatorname{MVI}(+)$ & MVI(-) & $\chi^{2}$ test & P-value \\
\hline Change of tumor size & & & 0.75 & 0.55 & & & 7.05 & 0.04 \\
\hline Conspicuous & 39 & 32 & & & 56 & 15 & & \\
\hline Inconspicuous & 53 & 56 & & & 74 & 35 & & \\
\hline Enhancement mode at arterial phase & & & 0.34 & 0.69 & & & 3.50 & 0.16 \\
\hline Inhomogeneous & 59 & 54 & & & 87 & 26 & & \\
\hline Homogeneous & 33 & 34 & & & 43 & 24 & & \\
\hline Enhancement form at arterial phase & & & 0.72 & 0.45 & & & 5.70 & 0.01 \\
\hline Irregular & 41 & 33 & & & 60 & 14 & & \\
\hline Regular & 51 & 55 & & & 70 & 36 & & \\
\hline Tortuous vessels & & & 0.13 & 0.56 & & & 5.70 & 0.01 \\
\hline Yes & 39 & 35 & & & 60 & 14 & & \\
\hline No & 53 & 53 & & & 70 & 36 & & \\
\hline Enhancement intensity at portal phase & & & 1.55 & 0.32 & & & 3.12 & 0.14 \\
\hline Low intensity & 65 & 57 & & & 93 & 29 & & \\
\hline High or equal intensity & 27 & 31 & & & 37 & 21 & & \\
\hline Extinction speed at portal phase & & & 2.31 & 0.42 & & & 3.54 & 0.03 \\
\hline Fast & 40 & 45 & & & 67 & 17 & & \\
\hline Low & 52 & 43 & & & 63 & 33 & & \\
\hline Extinction extent at portal phase & & & 1.75 & 0.33 & & & 3.88 & 0.14 \\
\hline Obvious & 65 & 57 & & & 92 & 29 & & \\
\hline None or slight & 27 & 31 & & & 38 & 21 & & \\
\hline
\end{tabular}

HCC, hepatocellular carcinoma; CEUS, contrast-enhancement ultrasound; H-MVD, high microvessel density; L-MVD, low microvessel density; MVI(+), microvessel invasion-positive group; MVI(-), microvessel invasion-negative group.

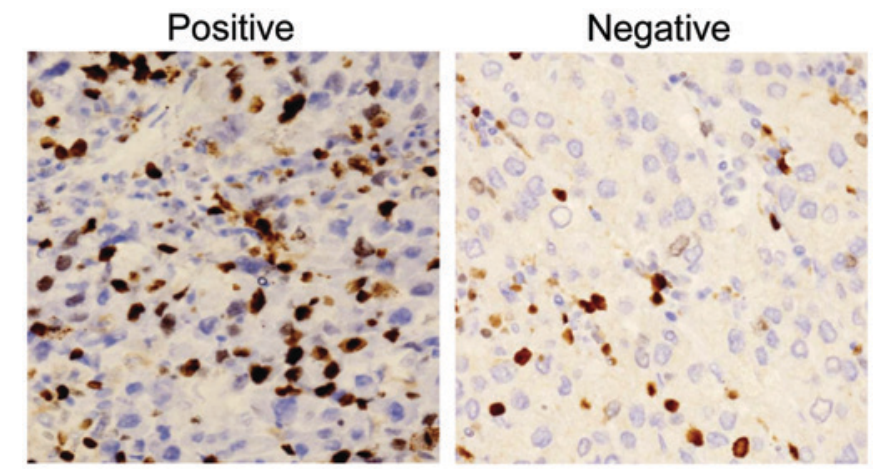

Figure 1. Different pathological expression levels of Ki-67 (ELPS staining, $\mathrm{x} 400$ ).

other parameters after CEUS and the immunohistochemical AFP (P>0.05, Table II).

There was no significant correlation between the change in tumor size, enhancement mode and form of tumor at arterial phase and other parameters after CEUS or the MVD level ( $P>0.05$, Table III). The incidence of positive MVI was high for those cases with obvious change in tumor size, irregular enhancement form of tumor at arterial phase, tortuous vessels inside and fast extinction at portal phase after CEUS $(\mathrm{P}<0.05$, Table III).

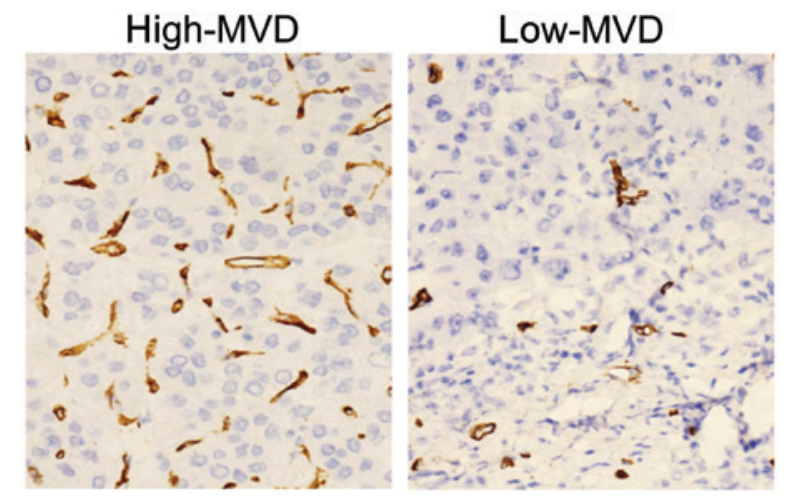

Figure 2. Different pathological expression levels of MVD (ELPS staining, $\mathrm{x} 400)$.

The tissue differentiation degree of tumor samples was lower for those cases with inhomogeneous enhancement and irregular enhancement form of tumor at arterial phase, tortuous vessels inside, low enhancement and fast extinction at portal phase after CEUS $(\mathrm{P}<0.05$, Table IV).

CEUS parameters vs. recurrence of liver cancer. The recurrence time was shorter for those cases with inhomogeneous enhancement of tumor at arterial phase in CEUS $(\mathrm{P}<0.05$, Table V and Fig. 4). 
Table IV. Comparison of CEUS parameters in tumor tissue differentiation HCC groups.

\begin{tabular}{|c|c|c|c|c|}
\hline Factors & Low/Medium-differentiated & Highly-differentiated & $\chi^{2}$ test & P-value \\
\hline Change of tumor size & & & 4.34 & 0.07 \\
\hline Conspicuous & 64 & 8 & & \\
\hline Inconspicuous & 96 & 22 & & \\
\hline Enhancement mode at arterial phase & & & 6.75 & 0.01 \\
\hline Inhomogeneous & 105 & 12 & & \\
\hline Homogeneous & 45 & 18 & & \\
\hline Enhancement form at arterial phase & & & 5.12 & 0.04 \\
\hline Irregular & 66 & 8 & & \\
\hline Regular & 84 & 22 & & \\
\hline Tortuous vessels & & & 7.13 & 0.03 \\
\hline Yes & 64 & 7 & & \\
\hline No & 86 & 23 & & \\
\hline Enhancement intensity at portal phase & & & 6.45 & 0.01 \\
\hline Low intensity & 102 & 13 & & \\
\hline High or equal intensity & 48 & 17 & & \\
\hline Extinction speed at portal phase & & & 6.83 & 0.00 \\
\hline Fast & 106 & 12 & & \\
\hline Low & 44 & 18 & & \\
\hline Extinction extent at portal phase & & & 4.27 & 0.04 \\
\hline Obvious & 68 & 9 & & \\
\hline None or slight & 82 & 21 & & \\
\hline
\end{tabular}

HCC, hepatocellular carcinoma; CEUS, contrast-enhancement ultrasound.

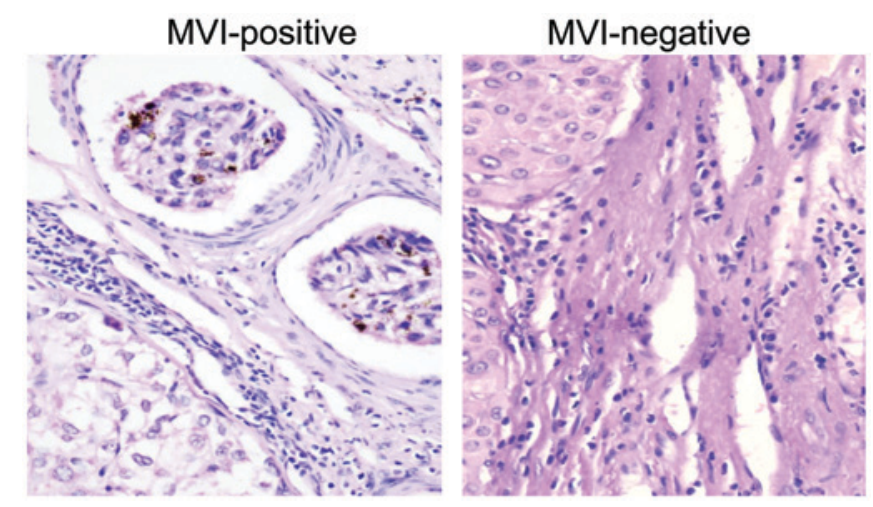

Figure 3. Different pathological expression levels of MVI (H\&E staining, $\mathrm{x} 400)$.

Logistic regression for prognostic factors of HCC. Logistic regression showed that the inhomogeneous enhancement inside at the arterial phase in CEUS was more helpful to suggest positive Ki-67 and early recurrence; irregular enhancement form at arterial phase suggested a higher risk of MVI; the low enhancement at portal phase might be a parameter suggesting low tissue differentiation degree $(\mathrm{P}<0.05$, Table VI).

\section{Discussion}

Approximately 740,000 people die from primary HCC around the world every year, and the patients in China account for

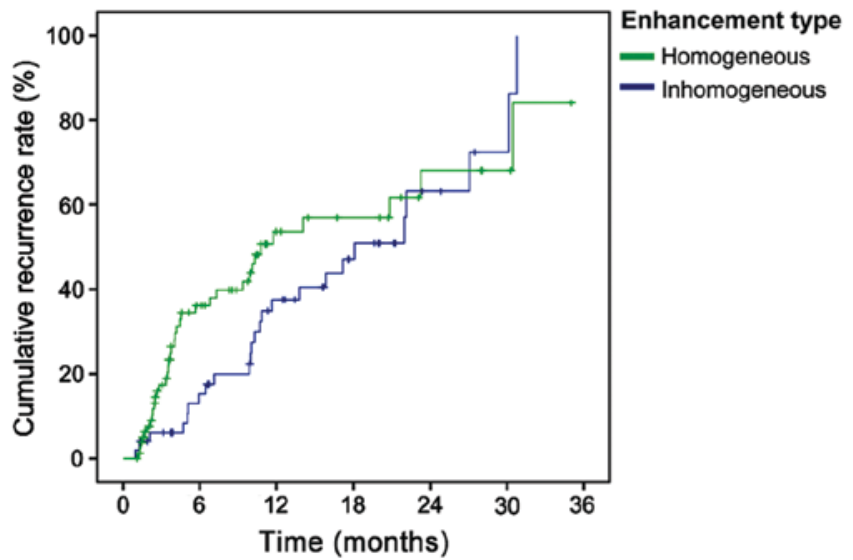

Figure 4. Recurrence rate of HCC under different enhancement at arterial phase.

more than $50 \%$ of the global total (2). The high postoperative recurrence rate of liver cancer is the main factor influencing the survival rate of patients with liver cancer. Early prediction of related risk factors to postoperative recurrence of liver cancer is of great clinical significance, and examining the postoperative recurrence as soon as possible and taking treatment measures timely can effectively improve the prognosis of patients with liver cancer (4).

The prognosis of HCC patients is determined by a variety of factors. Immunohistochemical Ki-67 is a rapid, simple and 
Table V. Comparison of CEUS parameters in different recurrence conditions of HCC groups.

\begin{tabular}{|c|c|c|c|c|c|}
\hline Factors & Early recurrence & Late recurrence & No recurrence & $\chi^{2}$ test & P-value \\
\hline Change of tumor size & & & & 1.90 & 0.43 \\
\hline Conspicuous & 26 & 8 & 38 & & \\
\hline Inconspicuous & 35 & 17 & 56 & & \\
\hline Enhancement mode at arterial phase & & & & 7.84 & 0.04 \\
\hline Inhomogeneous & 40 & 10 & 59 & & \\
\hline Homogeneous & 21 & 15 & 35 & & \\
\hline Enhancement form at arterial phase & & & & 5.87 & 0.07 \\
\hline Irregular & 28 & 7 & 42 & & \\
\hline Regular & 33 & 18 & 52 & & \\
\hline Tortuous vessels & & & & 4.30 & 0.38 \\
\hline Yes & 29 & 9 & 35 & & \\
\hline No & 32 & 16 & 59 & & \\
\hline Enhancement intensity at portal phase & & & & 4.12 & 0.30 \\
\hline Low intensity & 40 & 12 & 61 & & \\
\hline High or equal intensity & 21 & 13 & 33 & & \\
\hline Extinction speed at portal phase & & & & 5.13 & 0.24 \\
\hline Fast & 30 & 8 & 41 & & \\
\hline Low & 31 & 17 & 53 & & \\
\hline Extinction extent at portal phase & & & & 3.07 & 0.36 \\
\hline Obvious & 40 & 12 & 59 & & \\
\hline None or slight & 21 & 13 & 35 & & \\
\hline
\end{tabular}

HCC, hepatocellular carcinoma; CEUS, contrast-enhancement ultrasound.

Table VI. Logistic regression of HCC prognosis factors.

\begin{tabular}{|c|c|c|c|c|}
\hline Factors & r-value & Wald value & P-value & OR \\
\hline \multicolumn{5}{|l|}{$\mathrm{Ki}-67$} \\
\hline Inhomogeneous enhancement mode at arterial phase & 1.15 & 26.39 & NS & 2.78 \\
\hline \multicolumn{5}{|l|}{ MVI } \\
\hline Irregular enhancement form at arterial phase & 1.26 & 17.35 & NS & 3.10 \\
\hline \multicolumn{5}{|l|}{ Tumor tissue differentiation } \\
\hline Inhomogeneous enhancement mode at arterial phase & 1.20 & 7.03 & 0.02 & 3.25 \\
\hline Low intensity enhancement at portal phase & 1.55 & 12.39 & NS & 4.23 \\
\hline
\end{tabular}

HCC, hepatocellular carcinoma; MVI, microvessel invasion; OR, odds ratio. NS, not significant.

sensitive detection technique for proliferative activity of liver cancer cells, and its overexpression $(>10 \%)$ can predict the recurrence of liver cancer after surgical treatment, which is associated with the mortality of HCC patients (5-7). In this study, the change in lesion size, the enhancement mode and form inside lesion at arterial phase, and the enhancement intensity, extinction time and degree at portal phase were associated with the incidence of positive $\mathrm{Ki}-67$; and the logistic regression analysis showed that the inhomogeneous enhancement inside the lesion at arterial phase might be the best parameter to predict the $\mathrm{Ki}-67$ overexpression.
AFP is an important biological marker of liver cancer, whose quantification and immunohistochemistry is associated with the occurrence and development of liver cancer. In recent years, the studies show that AFP lacks sufficient sensitivity and specificity in the effective diagnosis and monitoring of liver cancer. There are few studies about the CEUS parameters and immunohistochemical level of AFP. The results of this study suggested that there was no obvious relationship between the immunohistochemistry AFP and CEUS parameters.

Tumor growth and metastasis depend on the growth of tumor angiogenesis, and MVD, as a sign of tumor angiogenesis 
degree, also suggests that it is closely related to the occurrence, development and metastasis of tumors $(11,12)$. Compared with liver cancer patients with low MVD, patients with high MVD tend to suffer from early postoperative recurrence. Some scholars studied the correlation between time intensity curve and MVD, and found that the peak intensity, intensity enhancement and area under the curve were positively correlated to the MVD count (13), but in this study, the change in tumor size, enhancement form at arterial phase and other parameters after CEUS had no significant relationship with MVD count.

MVI of liver cancer means that the cancer cells have the potential of intrahepatic metastasis and spread to the circulatory system $(14,15)$. In this study, it can be seen that the change in lesion size, enhancement form at arterial phase, tortuous vessels inside and extinction time at portal phase were related to MVI. Logistic regression analysis showed that irregular form at arterial phase was the most closely related to MVI. The irregular enhancement form of lesion at arterial phase is associated with the high incidence of MVI from both univariate and multivariate regression analysis, which may be the most valuable independent factor of predicting MVI.

Typical CEUS of HCC is characterized by high enhancement of lesion at arterial phase and low enhancement at portal phase and delayed phase, namely the so-called 'fast in and fast out' of contrast agent. However, the high-differentiated HCC may present the atypical imaging manifestations, such as the 'fast in and slow out' or 'slow in and slow out' of contrast agent (8-10). In this study, the enhancement mode and form of lesions at arterial phase, tortuous vessels, and the enhancement intensity, extinction time and degree at portal phase were correlated with the pathological tissue differentiation degree. Logistic regression analysis showed that the inhomogeneous enhancement inside lesions at arterial phase and low enhancement at portal phase were risk factors of low tissue differentiation degree. Compared with the inhomogeneous enhancement at arterial phase, low enhancement at portal phase is more dangerous. The study results showed that the proportion of high or equal enhancement at portal phase in high-differentiated HCC group was significantly higher than that in moderate- and low-differentiated group, and the reason may be that: i) high-differentiated HCC causes the retention of contrast agent due to the presence of neat bone trabeculatype cellular bundles and a large number of hepatic sinusoids; ii) with the increase of malignancy degree, the degree of differentiation becomes lower and the abnormal arteries are formed derived from the increase of abnormal arterial blood supply. Normal blood supply in hepatic artery and portal vein decreases, the duration of enhancement at portal phase declines and the enhancement intensity is lower; iii) there are many abnormal new vessels at the center or around the tumor, as well as abundant arteriovenous fistulas, thus shortening the duration of enhancement.

To sum up, this study investigated the relationship between the enhancement pattern and parameters of CEUS for HCC and the prognostic factors and clinical recurrence, and evaluated the biological behavior and prognosis of liver cancer from the perspective of pathology and ultrasonography, so as to provide a new prognosis evaluation index.

On the background of liver cirrhosis, there were significant differences in different enhancement modes and quantification parameters of contrast-enhancement ultrasound for HCC with different expression of Ki-67. The high incidence of positive Ki-67 may suggest poor prognosis. CEUS is helpful to predict the prognosis of HCC. The inhomogeneous enhancement at arterial phase may predict the proliferative activity and recurrence time of tumor cells; irregular enhancement form at arterial phase may indicate tumor MVI; and the low enhancement of tumor at portal phase may predict a lower degree of tissue differentiation and poor prognosis.

\section{Competing interests}

The authors declare that they have no competing interests.

\section{References}

1. Geschwind JF: Locoregional therapy for patients with hepatocellular carcinoma. Gastroenterol Hepatol (NY) 11: 698-700, 2015.

2. Gish RG: Hepatocellular carcinoma: Current questions and future directions. Gastroenterol Hepatol (NY) 11: 182-185, 2015.

3. Geschwind JF: Locoregional therapy for patients with hepatocellular carcinoma. Clin Adv Hematol Oncol 13: 660-662, 2015.

4. Janevska D, Chaloska-Ivanova V and Janevski V: Hepatocellular carcinoma: Risk factors, diagnosis and treatment. Open Access Maced J Med Sci 3: 732-736, 2015.

5. Tawfik O, Garimella R, Tancabelic J, Keighley J, Pinson D, Khan Q and Templeton K: Retrospective immunohistochemical study of VDR, RXR, Ki-67, and Bcl-2 expression in primary and metastatic osteosarcoma. J Clin Oncol 27: e21516, 2009.

6. Li W, Zhang G, Wang HL and Wang L: Analysis of expression of cyclin E, p27kip1 and Ki67 protein in colorectal cancer tissues and its value for diagnosis, treatment and prognosis of disease. Eur Rev Med Pharmacol Sci 20: 4874-4879, 2016.

7. Saponara M, Di BM, Lolli C, Derenzini E, Pantaleo MA, Santini D, Ceccarelli C, Catena F, Nannini M and Maleddu A: Evaluation of Ki-67 in gastrointestinal stromal tumor (GIST). J Clin Oncol 27: e21510, 2009.

8. Sirlin C: Imaging techniques for hepatocellular carcinoma. Gastroenterol Hepatol (NY) 11: 403-405, 2015.

9. Dong Y, Wang WP, Mao F, Fan M, Ignee A, Serra C, Sparchez Z, Sporea I, Braden B and Dietrich CF: Contrast enhanced ultrasound features of hepatic cystadenoma and hepatic cystadenocarcinoma. Scand J Gastroenterol 52: 365-372, 2017.

10. Haimerl M, Poelsterl S, Beyer LP, Wiesinger I, Niessen C, Stroszczynski C, Wiggermann P and Jung EM: Chronic liver disease: Quantitative MRI vs CEUS-based microperfusion. Clin Hemorheol Microcirc 64: 435-446, 2016.

11. Lekovic D, Gotic M, Skoda R, Beleslin-Cokic B, Milic N, Mitrovic-Ajtic O, Nienhold R, Sefer D, Suboticki T, Buac M, et al: Bone marrow microvessel density and plasma angiogenic factors in myeloproliferative neoplasms: Clinicopathological and molecular correlations. Ann Hematol 96: 393-404, 2017.

12. Zhang S, Zhang D, Yi S, Gong M, Lu C, Cai Y, Tang X and Zou L: The relationship of lymphatic vessel density, lymphovascular invasion, and lymph node metastasis in breast cancer: A systematic review and meta-analysis. Oncotarget 8: 2863-2873, 2017.

13. Poon RT, Ng IO, Lau C, Yu WC, Yang ZF, Fan ST and Wong J: Tumor microvessel density as a predictor of recurrence after resection of hepatocellular carcinoma: A prospective study. J Clin Oncol 20: 1775-1785, 2002.

14. Donat M, Alonso S, Pereira F, Ferrero E, Carrión L, AcinGándara D and Moreno E: Impact of histological factors of hepatocellular carcinoma on the outcome of liver transplantation. Transplant Proc 48: 1968-1977, 2016.

15. Feng LH, Dong H, Lau WY, Yu H, Zhu YY, Zhao Y, Lin YX, Chen J, Wu MC and Cong WM: Novel microvascular invasionbased prognostic nomograms to predict survival outcomes in patients after R0 resection for hepatocellular carcinoma. J Cancer Res Clin Oncol 143: 293-303, 2017.

This work is licensed under a Creative Commons Attribution-NonCommercial-NoDerivatives 4.0 International (CC BY-NC-ND 4.0) License. 